\title{
Emerging roles of steroid receptor coactivators in stromal cell responses
}

\author{
Aarti D Rohira, David M Lonard and Bert W O'Malley \\ Department of Molecular and Cellular Biology, Baylor College of Medicine, Houston, Texas, USA
}

Correspondence should be addressed to B W O'Malley: berto@bcm.edu

\begin{abstract}
Tissue parenchyma is the functional unit of an organ and all of the remaining cells within that organ collectively make up the tissue stroma. The stroma includes fibroblasts, endothelial cells, immune cells, and nerves. Interactions between stromal and epithelial cells are essential for tissue development and healing after injury. These interactions are governed by growth factors, inflammatory cytokines and hormone signaling cascades. The steroid receptor coactivator (SRC) family of proteins includes three transcriptional coactivators that facilitate the assembly of multi-protein complexes to induce gene expression in response to activation of many cellular transcription factor signaling cascades. They are ubiquitously expressed and are especially critical for the developmental function of steroid hormone responsive tissues. The SRCs are overexpressed in multiple cancers including breast, ovarian, prostate and endometrial cancers. In this review, we focus on the role of the SRCs in regulating the functions of stromal cell components responsible for angiogenesis, inflammation and cell differentiation.
\end{abstract}

\author{
Key Words \\ - steroid receptor \\ coactivators \\ - tissue stroma \\ - inflammation \\ - hormone signaling
}

\section{Tissue stroma}

Tissue stroma is defined as the connective tissue that supports tissue parenchyma. The parenchyma consists of the functional cellular unit of an organ while the extracellular matrix and support cells make up the tissue stroma. The main cell types comprising tissue stroma include fibroblasts, pericytes, nerves, endothelial cells, and immune cells. However, depending on tissue type, the stroma may include additional cell types such as adipocytes and mesenchymal stromal cells. Tissue stroma is essential for extracellular matrix production, blood vessel formation and immune surveillance.

\section{Fibroblasts}

Fibroblasts are the most common cells that make up the tissue stroma. They are mesenchymal in origin and are flat, spindle shaped cells with multiple processes extending from their cell body. Several cellular marker proteins such as vimentin, fibroblast specific protein 1 (FSP1) and fibroblast activating protein 1 (FAP1) have been used to identify fibroblast cell types, however, these genes are not uniquely expressed in fibroblasts (Krenning et al. 2010). Fibroblasts synthesize and secrete extracellular matrix components such as collagens, fibronectin and glycosaminoglycans that form the structural, adhesive, and ground support framework for a tissue. They play an essential role in wound healing and are the first responders that secrete chemokines and other factors in response to wounding, which then initiates an inflammatory cascade necessary for wound repair (Smith et al. 1997). Fibroblasts are not terminally differentiated cells and can be activated by a number of signals 
to proliferate and differentiate into myofibroblasts. Myofibroblasts are typically characterized by expression of smooth muscle actin ( $\alpha \mathrm{SMA})$. These cells play a vital role in wound healing and their continued activation can lead to pathological conditions such as tissue fibrosis (Kendall \& Feghali-Bostwick 2014).

\section{Endothelial cells}

Endothelial cells are mesodermal in origin and are characterized by several markers such as Von Willebrand factor (VWF), angiotensin converting enzyme (ACE) and CD31 to name a few. These cells form the inner lining of blood vessels and are essential for blood flow, oxygenation, and nutrient exchange within tissues as well as providing a barrier function and regulating the exchange of materials and immune cells within the tissue. Angiogenesis is a process through which endothelial cells give rise to new blood vessels within tissues during the repair of damaged or diseased organs; they also play an important role in signaling to immune cells during inflammation (Lingen 2001).

\section{Immune cells}

Immune cells are mediators of the host defense system and play a central role in detecting and eliminating pathogens. They are activated by inflammatory signals and go on to clear dead cells and debris to facilitate tissue repair. Based on their functions, immune cells are categorized into the innate or adaptive immune systems. The innate immune system serves as the first line of defense against foreign pathogens and consists of physical and chemical barriers that are non-specific. The innate immune system acts immediately to prevent the spread of foreign pathogens within the host. Cells such as macrophages and neutrophils are part of the innate immune system and serve as the first line of defense for eliminating pathogens (Lingen 2001). They set up inflammatory signaling programs in response to an infection and eliminate pathogens through phagocytosis. The adaptive immune system is the second line of host defense and functions by recognizing specific antigens on pathogens. $\mathrm{T}$ and $\mathrm{B}$ cells together comprise the adaptive immune system that function through cell-mediated and antibody responses that are specific to pathogen-related epitopes. Importantly, the adaptive immune system provides immunological memory that provides protection against subsequent infections by the same pathogen.

\section{Mesenchymal stromal cells}

More recently a new type of tissue stromal cell called a mesenchymal stromal cell was isolated from bone marrow, adipose and other tissues (Horwitz et al. 2006). These cells are spindle shaped and are thought to play a critical role within the bone marrow microenvironment. In vitro, these cells display multipotent differentiation capacity and can be differentiated into adipocytes, osteoblasts, and chondrocytes. Mesenchymal stromal cells are being investigated for potential therapeutic use as a cell-based treatment option for multiple human diseases.

\section{Tissue stroma in development, injury and repair}

Stromal cells are required for tissue development and tissue injury repair. The role of stromal cells in tissue injury repair has been characterized best using a skin wound healing model (Bainbridge 2013). In this model at the onset of wounding, endothelial cells bring about vasoconstriction to stop blood loss. Macrophages and neutrophils then migrate into the wound in response to pro-inflammatory signals where they help in the clearance of microbes and dead tissue within the wound bed. Next, fibroblasts within the wound proliferate and secrete collagen and other extracellular matrix components, supporting angiogenesis and wound closure. The endothelial cells respond to angiogenic cues generated by the fibroblasts and help establish new blood vessels at the wound site. Epithelial cells then proliferate and migrate over the wound bed matrix in a process termed 're-epithelialization'. In this way, the stromal components of the skin namely, immune cells, endothelial cells and fibroblasts work in concert to bring about wound repair and healing.

Tissue stromal cells also play vital roles in tissue development of various organs. This is best demonstrated in the mammary gland, where stromal fibroblasts and preadipocytes interact with breast epithelial cells and mediate breast development, postnatal ductal growth and ductal branching morphogenesis (Arendt et al. 2010). The mammary gland is composed of the epithelial parenchyma and two types of mesenchymal stroma which include fibroblasts and pre-adipocytes. Tissue transplantation studies have shown that when non-mammary epithelium is combined with mammary mesenchyme it results in mammary gland like ductal structures. Conversely, when mammary epithelium is combined with salivary 
mesenchyme, the resulting outgrowths closely resemble the salivary gland (Sakakura et al. 1976). Hence, the fibroblastic mesenchyme in the mammary gland is important for cell fate determination. The preadipocyte mesenchyme has been shown to be important for shaping the ductal branching structures and may inhibit the growth of the mammary epithelium. In another example, endometrial stromal fibroblasts in the uterus undergo a process of decidualization during pregnancy to form specialized decidual cells that are required for embryo implantation (Ramathal et al. 2010). The decidualization process occurs after ovulation during the mid-secretory phase of the menstrual cycle and the decidua is shed off in the absence of embryo implantation. This process is regulated by the sex steroid hormones progesterone and estrogen. Impairment in the process of decidualization leads to many pregnancy disorders such as infertility and miscarriages. Hence, the endometrial stromal cells have an essential role for the establishment and maintenance of pregnancy.

\section{Tissue stroma and disease}

While the tissue stroma is important for tissue repair process, deregulation in the stromal response can lead to pathological outcomes such as chronic inflammation and tissue fibrosis.

\section{Chronic inflammation}

An inflammatory reaction is triggered in the body in response to harmful stimuli or tissue injury. Damaged cells secrete chemical signals to help recruit immune cells such as leucocytes to the site of injury to clear out the harmful stimuli and help restore tissue homeostasis (Chen et al. 2018). This process is vital to human health. However, this acute inflammation can become deregulated and non-resolving thereby leading to chronic inflammation which causes tissue damage. Many chronic diseases such as cardiovascular disease, inflammatory bowel disease and arthritis are characterized by chronic inflammation. Chronic inflammation also can target and damage other organs such as the lungs, kidneys, and brain (Chen et al. 2018). Inflammatory cytokines secreted in response to tissue injury are key mediators of inflammation and are important for the recruitment of immune cells to the site of injury for tissue healing (Turner et al. 2014). Once the injury has been resolved, anti-inflammatory cytokines help inhibit inflammation. Multiple signaling pathways such as the NF-кB, MAPK and JAK-STAT pathways help regulate the expression of these cytokines (Chen et al. 2018). However, dysregulation of these signaling pathways leads to excessive production of pro-inflammatory cytokines which cause tissue damage. Tissue resident stromal cells such as fibroblasts also have been implicated in non-resolving chronic inflammation. Fibroblasts secrete cytokines and express adhesion molecules that are important for immune cell recruitment and retention at the injury site. Once debris has been cleared and injury resolved by the immune cells it is important for them to be cleared out from the injury site. However, misexpression of adhesion molecules and cytokines can retain immune cells within the tissue, resulting in chronic inflammation (Buckley et al. 2001, Van Linthout et al. 2014). Hence, the interactions between immune cells and fibroblasts are important to understand in order to guide the development of therapeutic interventions targeting chronic inflammation.

\section{Fibrosis}

Depending on the conditions and tissue context, fibrosis can be a pathological result of maladaptive wound healing. As mentioned previously, fibroblasts residing within an injury site undergo trans-differentiation in response to inflammatory cytokines into $\alpha \mathrm{SMA}$ expressing myofibroblasts (Klingberg et al. 2013). These myofibroblasts secrete abundant extracellular matrix components such as collagen to allow wound contracture and closure. This initial deposition of ECM for wound closure is required and clearly is beneficial for wound healing. However, this process can become deregulated resulting in an excessive deposition of ECM proteins which replace normal tissue parenchyma and lead to the formation of a fibrotic scar that causes functional organ damage (Wynn 2008). It has less deleterious effect in skin, but can be a problem within internal organs. Pulmonary, kidney and cardiac fibrosis are common fibrotic diseases that have no good therapeutic interventions. Hence, understanding mechanisms that cause tissue fibrosis is important for the development of new therapies.

\section{Cancer}

Recent studies have shed light on the role the stroma within the tumor microenvironment plays in tumor growth and resistance to therapy. The tumor microenvironment is composed of cancer associated fibroblasts (CAFs), blood vessels and immune cells such as tumor associated 
macrophages (TAMs), myeloid derived suppressor cells (MDSCs) and T regulatory cells (Tregs). The CAFs are the dominant cell type within the tumor microenvironment and are activated at the start of neoplasia (Foster et al. 2018). These activated CAFs produce ECM proteins, proteases and cytokines and are protumorigenic and proangiogenic. For instance, matrix metalloproteases (MMPs) help digest the basement membrane and allow the tumor epithelial cells to invade through the basement membrane to facilitate metastasis. Angiogenic signals produced by the tumor cells and CAFs give rise to new leaky vasculature that promotes metastasis and allows invasion of immune cells. These immune cells include Tregs and MDSCs which help suppress the immune response and allow the proliferation of the tumor cells. Emerging evidence also has shed light on the role of the tumor microenvironment and stromal cells in promoting resistance to therapeutic interventions (Wu \& Dai 2017). TAMs have been shown to promote chemoresistance to drugs such as paclitaxel, gemcitabine, and doxorubicin in vitro and in vivo. MDSCs also mediate resistance to chemotherapy drugs such as cyclophosphamide, sunitinib and anthracycline. Additionally, both TAMs and MDSCs promote resistance to anti-VEGF therapy. Continued investigations into the interactions between the tumor stromal and epithelial cells are therefore important for the identification of new therapeutic targets.

\section{Tissue stroma-epithelium paracrine signaling}

Paracrine signaling between stromal and epithelial cells by cytokines, growth factors and hormones have been shown to regulate the crosstalk between tissue stroma and parenchyma. Previous studies have shown that steroid hormones such as estrogens, progestins and androgens play key roles in regulating stromal-epithelial interactions during tissue development and healing. Estrogen signaling is important for breast and prostate development (Mueller et al. 2002) and wound healing (Horng et al. 2017) and has beneficial effects on tissue vasculature (White 2002), while progesterone hormone signaling is required for endometrial cell decidualization (Dunn et al. 2003). One of the most well characterized examples of tissue paracrine signaling is during the process of endometrial decidualization and embryo implantation. The decidualization reaction is essential for successful implantation of the embryo. After embryo implantation, the decidua further develops to aid placentation. Paracrine signaling between the uterine stroma and epithelium plays an important role in making the uterus receptive for implantation. Studies using uterine tissue recombination and conditional deletions of estrogen and progesterone receptors have found that stromal ER signaling is required for uterine epithelial cell proliferation (Pawar et al. 2014). One proposed mechanism by which this occurs is through paracrine signaling via stromal ER induced expression of insulin growth factor (IGF) that signals through IGF-1 receptors on the epithelium. Indian hedgehog (IHH), leukemia inhibitory factor (LIF) and heparin-binding EGF (Hb-EGF) are other paracrine effectors that regulate uterine receptivity for embryo implantation. Epithelial PR regulates IHH production which acts by regulating stromal COUP-TFII signaling to promote decidualization. In another example, conditional deletion of the $E R$ gene in the uterine epithelial compartment leads to loss of LIF production. Furthermore, the transcription factor signal transducer and activator of transcription 3 (STAT3) acts downstream of LIF in the epithelium to induce stromal cell proliferation by secretion of Hb-EGF. Mice lacking uterine epithelial specific STAT3 show decreased phosphorylation of the EGF receptor in the uterine stroma after implantation. Importantly, exogenous administration of EGF family effectors rescues the stromal cell proliferation defects in the mice lacking STAT3 in the uterine epithelium. Together, these results highlight the importance of steroid hormone signaling in regulating paracrine signaling between the tissue stroma and epithelial cellular compartments.

\section{Steroid hormone signaling}

Steroid hormones signal through nuclear hormone receptors such as the estrogen receptor, progesterone receptor, and androgen receptor (Sever \& Glass 2013). These nuclear hormone receptors may be present in the nucleus or the cytoplasm. The receptors contain a $\mathrm{N}$-terminal domain that is less conserved among the different receptors, a DNA binding domain that mediates binding of the hormone bound receptor to the hormone response element within the target gene enhancers and promoters, a hinge region and a C-terminal ligand binding domain (Mangelsdorf et al. 1995). Nuclear hormone receptors that are present in the cytoplasm contain a nuclear localization signal (NLS) within the hinge region and in the absence of hormone, they bind to heat shock proteins such as hsp90 which obscures the NLS within the hinge region (Echeverria \& Picard Didier 2010). Upon hormone binding, the receptor conformation is 
changed which releases it from the chaperone proteins and exposes the NLS. This allows translocation of the hormone bound receptor to the nucleus where it can bind DNA and activate transcription. For nuclear hormone receptors that are present within the nucleus, hormone binding facilitates dimer formation and DNA binding. Once stimulated with hormone and bound to DNA these nuclear hormone receptors interact with and assemble transcriptional coactivators and corepressors that are required to facilitate gene transcription. The steroid receptor coactivator (SRC) proteins are the major family of fundamental transcriptional coactivators that bind to ER, PR and AR to facilitate gene transcription that we will discuss in more detail below.

\section{Steroid receptor coactivators}

The steroid receptor coactivators (SRCs) consist of a family of three proteins. They function as transcriptional coactivator platform proteins that form a base for the assembly of multi-subunit protein complexes on transcription factors (TFs) at promoters to stimulate transcription (Xu et al. 2009). The three family members SRC-1, SRC-2 and SRC-3 share about 60\% sequence homology with each other. They contain three main functional domains consisting of the basic helix-loophelix (bHLH)/Per/Arnt/Sim (bHLH-PAS) domain, the nuclear receptor interaction domain (RID) that contains two LXXL motifs (where $\mathrm{X}$ is any amino acid) and, two C-terminal activation domains termed AD1 and AD2. While there is some functional redundancy among the three family members, they also have important and distinct roles. They are the main coactivators required for the action of nuclear hormone receptors such as estrogen (ER), androgen (AR), progesterone (PR), and glucocorticoid receptors (GR). They also serve as coactivators for other nuclear receptors such as the retinoic acid receptor (RAR), the peroxisome proliferator-activated receptor- $\gamma(\operatorname{PPAR} \gamma)$ as well as other transcription factors including NF-кB.

While whole body knockout of any of the three SRCs do not result in embryonic lethality, studies have shown clear defects within the reproductive and hormone responsive tissues in the knockout animals (Dasgupta et al. 2014).

\section{SRCs and reproduction}

SRC-1 knockout mice show a significant decrease in uterine, prostate, testes and mammary tissue growth in response to hormone stimulation with no overt defects in viability and fertility. SRC-1 is also the major coactivator for ER and PR within the uterus and SRC-1 knockout leads to a $60 \%$ decrease in uterine growth. In contrast, SRC-2 knockout mice are hypofertile as a result of substantial reproductive tissue defects. Male SRC-2 knockout mice show defective spermatogenesis and testicular degeneration while female SRC-2 knockout mice have major defects in uterine decidual response in addition to placental hypoplasia and strongly impaired embryo implantation. SRC-2 is also important for progesterone-induced mammary ductal branching and alveologenesis. SRC-3 knockout mice display abnormal reproductive physiology, postnatal growth retardation, and delayed puberty. SRC-3 functions as the major coactivator for ER and PR in mammary luminal epithelial cells and loss of SRC-3 leads to reduced mammary ductal branching and alveologenesis. Collectively, all three SRCs are important for tissue development.

\section{SRCs and energy metabolism}

Global knockouts of the SRC family members in mice have highlighted the importance of the steroid receptor coactivators in regulating energy metabolism (Dasgupta et al. 2014). Whole body knockout of SRC-1 in mice causes an increased risk of obesity due to decreased energy expenditure. SRC-1 null mice also display hypoglycemia due to impaired hepatic gluconeogenesis and SRC-1 directly regulates gene expression of key rate limiting enzymes such as pyruvate carboxylase, phosphoenolpyruvate carboxykinase and fructose-1,6bisphosphatase involved in gluconeogenesis. Conversely, SRC-2 null mice are protected from high fat diet induced obesity and demonstrate increased insulin sensitivity, higher lipolysis, and decreased fat absorption. SRC-2 null mice also exhibit phenotypes similar to Von Gierke's disease due to reduced hepatic glucose release as a result of decreased expression of the glucose 6 phosphatase enzyme. Additionally, SRC-2 also plays an important role in coordinating whole-body energy homeostasis. It does this by stimulating fatty acid absorption from the gut through coactivation of farnesoid X receptor (FXR) to increase expression of bile salt export pump (BSEP). In contrast, knockout of SRC-3 in mice resembles human CACT metabolic myopathy (York et al. 2012). SRC-3 in skeletal muscle regulates expression of the long-chain fatty acid transporter, carnitine/acylcarnitine translocase (CACT) and loss of SRC-3 leads to accumulation of long chain acyl carnitines in skeletal muscle. Thus, all three SRCs regulate different aspects of energy metabolism. 


\section{SRCs and cancer}

All three SRCs also have been shown to promote cancer and metastasis in hormone dependent cancers (Rohira \& Lonard 2017). SRC-1 expression correlates with Her2 and c-myc expression and reduced disease-free survival in ER negative breast cancer. SRC-1 also promotes breast cancer cell invasion and migration by regulating expression of genes important for epithelial to mesenchymal transition (EMT) and is required for breast cancer metastasis. Indeed, knockout of SRC-1 in a PyMT mouse model for breast cancer did not affect tumor initiation and growth but significantly reduced metastasis to the lung. SRC- 1 also is overexpressed in endocrine and aromatase inhibitor resistant breast tumors and plays a role in the development of chemo-resistance. In addition to breast cancer, SRC-1 mRNA expression is significantly upregulated in endometrial cancer. SRC-1 expression also is elevated in androgen-dependent prostate cancer and is associated with increased tumor aggressiveness. SRC-2 is amplified in prostate cancer and increased expression is associated with higher metastasis and recurrence in prostate cancer patients. SRC-2 loss causes inhibition of prostate cancer cell growth and metastasis and SRC-2 promotes prostate cancer metastasis by markedly regulating cancer cell metabolism. SRC-2 mRNA level is also increased in endometrial cancer. Conversely, SRC-2 functions as a tumor suppressor for chemical carcinogen induced liver cancer. SRC-3 is overexpressed and/or amplified in breast cancer and is associated with decreased disease-free survival. SRC-3 is important for breast tumor growth and metastasis. Overexpression of SRC-3 in a MMTV mouse model induces spontaneous tumor formation in the mammary gland. Additionally, SRC-3 increases lung, liver, prostate and ovarian tumor growth. SRC-3 also increases lung cancer metastasis and ovarian cancer cell motility and migration. With their important roles in promoting tumor cell growth and metastasis the SRCs serve as a new class of targets for therapeutic development. Indeed, small molecule compounds (SI-2/SI-12) that repress SRC transcriptional activity are capable of reducing breast (Song et al. 2016), thyroid (Lee et al. 2020) and pancreatic (Song et al. 2019) tumor growth.

\section{SRCs in tissue stroma}

The SRC proteins are ubiquitously expressed in multiple cell types of the tissue stroma such as fibroblasts, endothelial and immune cells and all three SRCs regulate cell-autonomous and paracrine effects that influence stromal-epithelial cell interactions.

\section{SRC-1 is important for angiogenesis and endometrial stromal cell decidualization}

The vasoprotective function of estrogen (Orshal \& Khalil 2004) is demonstrated by reduced neointima formation and inhibition of neointimal cell proliferation after carotid artery ligation in mice. Estrogen signals through ER complexed with SRCs. The SRC-1 protein is expressed in endothelial and vascular smooth muscle cells in mice. SRC-1 knockout mice that have been ovariectomized show increased neointimal formation after carotid artery ligation with estrogen treatment compared to WT mice (Yuan \& Xu 2007). Additionally, SRC-1 knockout mice display increased proliferation of endothelial, vascular smooth muscle and neointimal cells compared to WT mice. Decreased SRC-1 expression also reduces endothelial cell migration and tube formation in vitro (Zhang \& Shi 2019). Hence, SRC-1 function in endothelial and vascular smooth muscle cells is important for the vasoprotective function of estrogen.

SRC-1 also supports the steroid hormone response in endometrialstromalcelldecidualization(Szwarcetal.2014). Estrogen and progesterone regulate the ability of the uterus to support pregnancy. Before implantation, progesterone stimulates endometrial stromal cell proliferation. At the time of implantation, endometrial stromal cells undergo differentiation to form decidual cells that are in direct contact with the embryo. The decidual response can be stimulated in mice by treating ovariectomized mice with a high dose of progesterone and a low dose of estrogen followed by mechanical traumatization (scratch). SRC-1 knockout mice in this model show only a partial response to decidual stimulation compared to WT mice (Xu et al. 1998). Additionally, endometrial stromal cells also express a proteolytic cleaved isoform of SRC-1 that is produced as a result of increased MMP9 activity during endometriosis (Han et al. 2012). This cleaved SRC-1 isoform is present in the cytoplasm and nucleus and plays a role in protecting endometrial epithelial cell apoptosis in the absence of paracrine endometrial stromal cell signaling.

\section{Direct and paracrine effects of SRC-2 in endothelial, endometrial and bone marrow stromal cells}

SRC-2 also regulates the endometrial stromal cell decidualization process in response to steroid hormone stimulation. Like SRC-1 knockout mice, SRC-2 knockout mice show a significant reduction in decidual response 
compared to WT mice when treated with a high dose of progesterone and a low dose of estrogen followed by mechanical traumatization of the uterus (Jeong et al. 2007). Proliferation of endometrial stromal cells is a key event before decidualization that is regulated by progesterone. SRC-2 drives this progesterone regulated proliferation by accelerating cellular glycolytic flux by upregulating the expression of the enzyme 6-phosphofructo-2-kinase/ fructose-2,6-bisphophatase 3 (PFKFB3) (Kommagani et al. 2013). This increase in glycolytic flux is necessary to provide energy and needed building biomolecules for rapid cellular proliferation. Indeed, acute treatment of mice with a small molecule inhibitor of PFKFB3 has been shown to suppress the ability of the animals to mount an endometrial decidual response. Furthermore, microarray analysis from SRC-2 depleted uterine tissue after acute progesterone treatment revealed that SRC- 2 is required for the induction of the progesterone transcriptional program. SRC-2 induced transcription factor 23 (TCF23) to modulate progesterone induced decidualization of endometrial stromal cells (Kommagani et al. 2014). Total RNA-seq from endometrial stromal cells undergoing in vitro decidualization after knockdown of SRC-2 expression revealed a connection between retinoid transport and SRC-2 signaling. Integrating RNA-seq and ChIP-seq data identified stimulated by retinoic acid 6 (STRA6) as an important target gene of progesterone and SRC-2 signaling (Szwarc et al. 2018). Together, SRC-2 has been shown to function as an important coactivator that can regulate progesterone receptor-induced transcriptional programs during endometrial stromal cell decidualization.

SRC-2 also regulates the differentiation of bone marrow stromal cells into adipocytes. The loss of SRC-2 expression in mice resulted in increased bone mass and increased trabecular bone volume. SRC-2 knockout mice also showed an increase in the number of osteoblasts and a decrease in the number of adipocytes within the bone marrow. Since SRC-2 functions as a transcriptional coactivator for PPAR $\gamma$ target genes, these effects can be attributed to a partial PPAR $\gamma$ resistance phenotype (Mödder et al. 2009). Mesenchymal stromal cells isolated from the bone marrow can be differentiated into osteoblasts or adipocytes in vitro. Bone marrow stromal cells isolated from SRC-2 knockout mice and cultured in osteoblastic medium displayed increased osteoblast colony forming units compared to WT mice. This effect was further enhanced in the presence of the PPAR $\gamma$ ligand rosiglitazone with stromal cells from SRC-2 knockout mice resulting in three times more osteoblast colony forming units compared to WT cells. Loss of SRC-2 reduced adipogenesis in bone marrow stromal cells and reduced expression of PPAR $\gamma$ target genes such as adiponectin and aP2 (fatty acid binding protein 2). Chromatin immunoprecipitation analysis found an increase in SRC-2 occupancy at the adiponectin PPRE (peroxisome proliferator response element) following rosiglitazone treatment in WT but not SRC-2 knockout bone marrow stromal cells. SRC-2 thus functions as a transcriptional coactivator mediating PPAR $\gamma$ effects on adipogenic genes in bone marrow stromal cells.

SRC-2 also has been shown to be an important mediator of paracrine signaling between cardiomyocytes and endothelial cells during pressure overload injury in the heart (Suh et al. 2017). In vitro, human umbilical vein endothelial cells (HUVECs) treated with conditioned media from cardiomyocyte-specific SRC-2 knockout cells displayed reduced proliferation and tube formation ability and this inhibition was rescued by treatment with exogenous vascular endothelial growth factor (VEGF). Furthermore, SRC-2 coactivated GATA-binding protein 4 (GATA-4) and hypoxia inducible factor (HIF)- $1 \alpha$ and $-2 \alpha$ in the cardiomyocytes to regulate the VEGF expression and secretion. In this way SRC-2 mediated regulation of VEGF expression in cardiomyocytes stimulates endothelial cell proliferation and tube formation.

\section{SRC-3 regulates endothelial and immune cell functions}

SRC-3 is highly expressed in endothelial cells and vascular smooth muscle cells. Similar to SRC-1, SRC-3 is also required for estrogen induced vascular repair after injury. SRC-3 knockout mice displayed a deficiency in neointimal inhibition and increased cell proliferation after estrogen treatment in ovariectomized mice after carotid artery ligation (Yuan et al. 2002). In vitro studies have shown that decreased SRC-3 expression in HUVECs reduced tube formation on matrigel and blocked their barrier function (Al-Otaiby et al. 2012). SRC-3 also is important for endothelial cell migration and proliferation. Further explanation for the molecular action of SRC-3 mediated regulation of endothelial cell migration and tube formation came from a study by Wang et al. They found that extracellular signal-regulated kinase 3 (ERK3) signaling in endothelial cells upregulated the interaction of SRC-3 and CREB-binding protein (CBP) with the transcription factor $\mathrm{Sp} 1$, leading to the upregulation of vascular endothelial growth factor receptor 2 (VEGFR2) expression (Wang et al. 2014). This increased expression of VEGFR2 in endothelial cells promoted HUVEC proliferation, migration, and tube formation. In addition 
to endothelial cell migration, SRC-3 also regulated the migration of fibroblasts (Al-Otaiby et al. 2012).

SRC-3 function also has been shown to be important for tissue repair. Complete healing of skin wounds in SRC-3 knockout mice is impaired compared to WT controls (Al-Otaiby et al. 2012). Histological analysis of these mice showed reduced neoangiogenesis and decreased infiltration of immune cells, namely macrophages and monocytes, in skin wounds. SRC-3 also regulates paracrine signaling between endothelial cells and tissue epithelial cells through modulation of fibroblast growth factor (FGF) signaling. FGF10 ligand produced by stromal cells that acts on epithelial cell FGFR2b is known to be important in wound re-epithelialization and angiogenesis (Zhang et al. 2006, Gurtner et al. 2008). Expression of FGF10 and FGFR2b were both reduced in skin wounds in SRC-3 heterozygous mice, again highlighting the role of SRC-3 in regulating paracrine signaling within tissue stroma.

SRC-3 regulates inflammatory signals within immune cells via suppression of cytokine release/production. Consequently, SRC-3 knockout mice are susceptible to endotoxic shock after lipopolysaccharide (LPS) treatment as a result of increased production of proinflammatory cytokines including tumor necrosis factor alpha (TNF $\alpha$ ), interleukin 1 beta (IL-1 $\beta$ ), and interleukin 6 (IL-6) (Yu et al. 2007). While mRNA levels of TNF $\alpha$ and IL-1 $\beta$ were unchanged in peritoneal macrophages between WT and SRC-3 knockout mice after LPS treatment, protein levels of
TNF $\alpha$ and IL- $1 \beta$ were increased in SRC- 3 knockout mice. This led to the discovery of a new role for SRC-3 as a 'translational repressor' of inflammation. SRC-3 brings about translation repression by interacting with the RNA binding proteins, $\mathrm{T}$ cell intracellular Ag-1 (TIA-1) and T cell intracellular Ag-1 related (TIAR), promoting their association with the AU rich regions within the TNF $\alpha$ mRNA. SRC-3 has been shown to regulate phagocytosis in macrophages and neutrophils. In E. coli induced peritonitis, SRC-3 expression in peritoneal macrophages enhanced bacterial clearance (Chen et al. 2010). Through its transcriptional coactivation function, SRC-3 upregulated the expression of the antioxidant enzyme, catalase and the macrophage surface receptor, SR-A which led to increased bacterial phagocytosis and decreased macrophage apoptosis. Collectively, SRC-3 function is important for endothelial cell angiogenesis and wound healing, fibroblast migration, and inflammatory signaling in immune cells.

\section{Conclusions}

Tissue stroma carries out essential functions in tissue development and tissue repair after injury. Research in the past few years has highlighted the pathological role of the stroma in maladaptive wound healing, chronic inflammation, tissue fibrosis and in diseases such as cancer. The tumor stroma has been shown to promote tumor cell proliferation, migration, and metastasis. Cancer

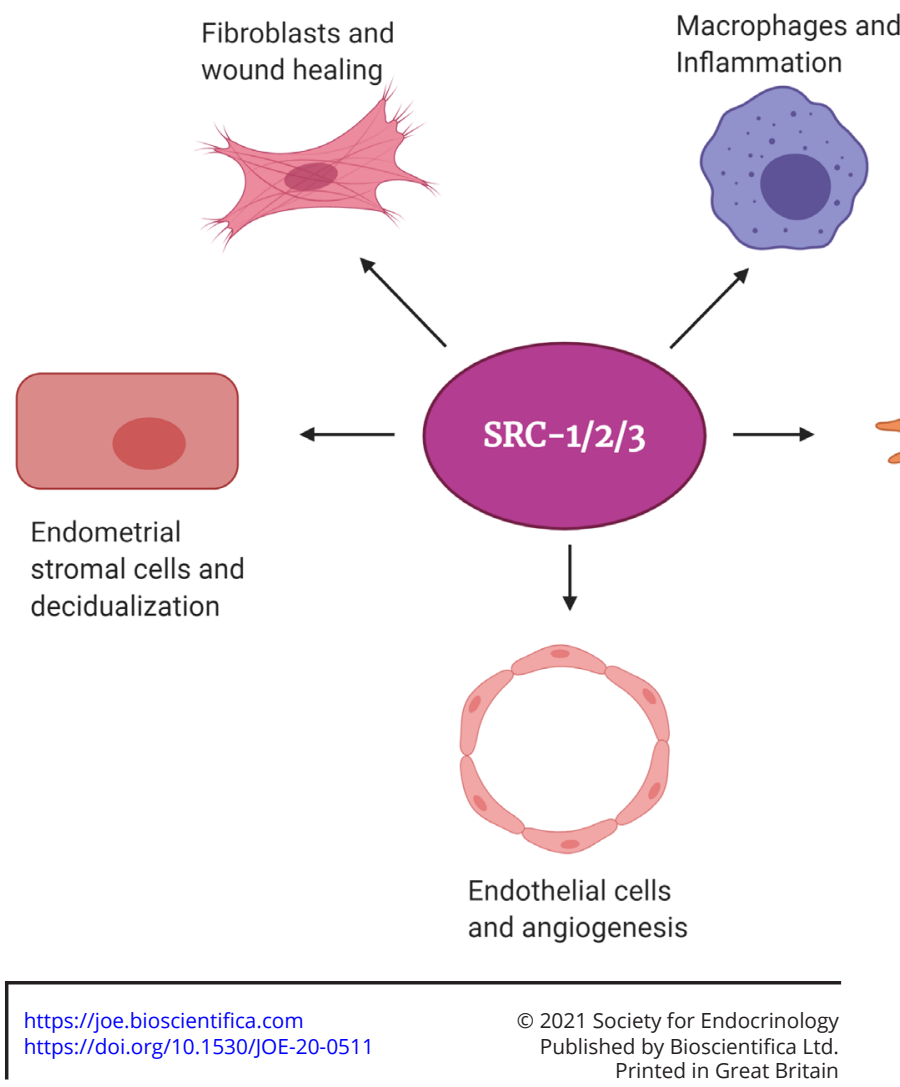

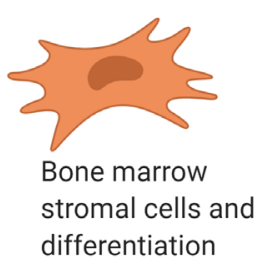

Figure 1

The steroid receptor co-activator (SRC) proteins have important roles in regulating stromal cell functions. https://joe.bioscientifica.com https://doi.org/10.1530/JOE-20-051 Printed in Great Britain 
associated fibroblasts promote tumor growth while tumor associated macrophages create an immunosuppressive microenvironment allowing the tumor to proliferate and resist therapy (Arendt et al. 2010). Efforts are currently underway to understand the crosstalk between the tumor stroma and cancer cells within the tumor microenvironment in order to identify and design more effective therapies.

Conversely, the tissue stroma also is important for normal tissue healing as evidenced by the stromal cells in the skin that are essential mediators of the wound healing response. Efforts are also currently underway to determine the mechanisms underlying tissue fibrosis in order to develop treatments for fibrotic diseases such as lung and kidney fibrosis. The SRCs are ubiquitously expressed in stromal cells and have been shown to have important regulatory roles in mediating stromal cell functions (Fig. 1). More recent work in our lab has shown that an SRC stimulator functions through cardiac fibroblasts and immune cells to protect cardiac function after a myocardial infarction (Mullany et al. 2020). In conclusion, this review highlights the importance of further examining the role of the SRCs in stromal responses in normal and pathologic physiology, tissue healing, and oncogenesis to help develop new therapeutic strategies.

\section{Declaration of interest}

The authors hold equity in Coactigon, Inc. which is developing steroid receptor coactivator inhibitors for clinical use.

\section{Funding}

This work was supported by grants HD07857 and HD 8818 from the National Institutes of Health.

\section{References}

Al-Otaiby M, Tassi E, Schmidt MO, Chien CD, Baker T, Salas AG, Xu J, Furlong M, Schlegel R, Riegel AT, et al. 2012 Role of the nuclear receptor coactivator AIB1/SRC-3 in angiogenesis and wound healing. American Journal of Pathology 180 1474-1484. (https://doi. org/10.1016/J.AJPATH.2011.12.032)

Arendt LM, Rudnick JA, Keller PJ \& Kuperwasser C 2010 Stroma in breast development and disease. Seminars in Cell and Developmental Biology 21 11-18. (https://doi.org/10.1016/J.SEMCDB.2009.10.003)

Bainbridge P 2013 Wound healing and the role of fibroblasts. Journal of Wound Care 22 407-408, 410-412. (https://doi.org/10.12968/ jowc.2013.22.8.407)

Buckley CD, Pilling D, Lord JM, Akbar AN, Scheel-Toellner D \& Salmon M 2001 Fibroblasts regulate the switch from acute resolving to chronic persistent inflammation. Trends in Immunology 22 199-204. (https:// doi.org/10.1016/s1471-4906(01)01863-4)
Chen Q, Chen T, Xu Y, Zhu J, Jiang Y, Zhao Y, Xu J \& Yu C 2010 SRC-3 is required for clearing bacteria and repressing inflammatory response in Escherichia coli-induced septic peritonitis. Journal of Immunology 185 5444-5452. (https://doi.org/10.4049/JIMMUNOL.0903802)

Chen L, Deng H, Cui H, Fang J, Zuo Z, Deng J, Li Y, Wang X \& Zhao L 2018 Inflammatory responses and inflammation-associated diseases in organs. Oncotarget 9 7204-7218. (https://doi.org/10.18632/ ONCOTARGET.23208)

Dasgupta S, Lonard DM \& O'Malley BW 2014 Nuclear receptor coactivators: master regulators of human health and disease. Annual Review of Medicine 65 279-292. (https://doi.org/10.1146/annurev-med-051812-145316)

Dunn CL, Kelly RW \& Critchley HO 2003 Decidualization of the human endometrial stromal cell: an enigmatic transformation. Reproductive Biomedicine Online 7 151-161. (https://doi.org/10.1016/S14726483(10)61745-2)

Echeverria PC \& Picard Didier D 2010 Molecular chaperones, essential partners of steroid hormone receptors for activity and mobility. Biochimica et Biophysica Acta 1803 641-649. (https://doi. org/10.1016/j.bbamcr.2009.11.012)

Foster DS, Jones RE, Ransom RC, Longaker MT \& Norton JA 2018 The evolving relationship of wound healing and tumor stroma. JCI Insight 3 e99911. (https://doi.org/10.1172/jci.insight.99911)

Gurtner GC, Werner S, Barrandon Y \& Longaker MT 2008 Wound repair and regeneration. Nature $\mathbf{4 5 3}$ 314-321. (https://doi.org/10.1038/ nature07039)

Han SJ, Hawkins SM, Begum K, Jung SY, Kovanci E, Qin J, Lydon JP, DeMayo FJ \& O'Malley BW 2012 A new isoform of steroid receptor coactivator-1 is crucial for pathogenic progression of endometriosis. Nature Medicine 18 1102-1111. (https://doi.org/10.1038/nm.2826)

Horng HC, Chang WH, Yeh CC, Huang BS, Chang CP, Chen YJ, Tsui KH \& Wang PH 2017 Estrogen effects on wound healing. International Journal of Molecular Sciences 18 2325. (https://doi.org/10.3390/IJMS18112325)

Horwitz EM, Andreef M \& Frassoni F 2006 Mesenchymal stromal cells. Current Opinion in Hematology 13 419-425. (https://doi. org/10.1097/01.MOH.0000245697.54887.6F)

Jeong JW, Lee KY, Han SJ, Aronow BJ, Lydon JP, O’Malley BW \& DeMayo FJ 2007 The p160 steroid receptor coactivator 2, SRC-2, regulates murine endometrial function and regulates progesteroneindependent and -dependent gene expression. Endocrinology 148 4238-4250. (https://doi.org/10.1210/en.2007-0122)

Kendall RT \& Feghali-Bostwick CA 2014 Fibroblasts in fibrosis: novel roles and mediators. Frontiers in Pharmacology 5 123. (https://doi. org/10.3389/fphar.2014.00123)

Klingberg F, Hinz B \& White ES 2013 The myofibroblast matrix: implications for tissue repair and fibrosis. Journal of Pathology 229 298-309. (https://doi.org/10.1002/path.4104)

Kommagani R, Szwarc MM, Kovanci E, Gibbons WE, Putluri N, Maity S, Creighton CJ, Sreekumar A, DeMayo FJ, Lydon JP, et al. 2013 Acceleration of the glycolytic flux by steroid receptor coactivator- 2 is essential for endometrial decidualization. PLoS Genetics 9 e1003900. (https://doi.org/10.1371/JOURNAL.PGEN.1003900)

Kommagani R, Szwarc MM, Kovanci E, Creighton CJ, O’Malley BW, DeMayo FJ \& Lydon JP 2014 A murine uterine transcriptome, responsive to steroid receptor coactivator-2, reveals transcription factor 23 as essential for decidualization of human endometrial stromal cells. Biology of Reproduction 90 75. (https://doi.org/10.1095/ biolreprod.114.117531)

Krenning G, Zeisberg EM \& Kalluri R 2010 The origin of fibroblasts and mechanism of cardiac fibrosis. Journal of Cellular Physiology 225 631-637. (https://doi.org/10.1002/JCP.22322)

Lee WK, Kim WG, Fozzatti L, Park S, Zhao L, Willingham MC, Lonard D, O'Malley BW \& Cheng SY 2020 Steroid receptor coactivator-3 as a target for anaplastic thyroid cancer. Endocrine-Related Cancer $\mathbf{2 7}$ 209-220. (https://doi.org/10.1530/ERC-19-0482)

Lingen MW 2001 Role of leukocytes and endothelial cells in the development of angiogenesis in inflammation and wound healing. https://joe.bioscientifica.com

https://doi.org/10.1530/JOE-20-0511
(C) 2021 Society for Endocrinology Published by Bioscientifica Ltd. Printed in Great Britain 
Archives of Pathology and Laboratory Medicine 125 67-71. (https://doi. org/10.1043/0003-9985(2001)125<0067:ROLAEC >2.0.CO;2)

Mangelsdorf DJ, Thummel C, Beato M, Herrlich P, Schütz G, Umesono K, Blumberg B, Kastner P, Mark M, Chambon P, et al. 1995 The nuclear receptor superfamily: the second decade. Cell 83 835-839. (https:// doi.org/10.1016/0092-8674(95)90199-x)

Mödder UI, Monroe DG, Fraser DG, Spelsberg TC, Rosen CJ, Géhin M, Chambon P, O'Malley BW \& Khosla S 2009 Skeletal consequences of deletion of steroid receptor coactivator-2/transcription intermediary factor-2. Journal of Biological Chemistry 284 18767-18777. (https://doi. org/10.1074/jbc.M109.000836)

Mueller SO, Clark JA, Myers PH \& Korach KS 2002 Mammary gland development in adult mice requires epithelial and stromal estrogen receptor $\alpha$. Endocrinology 143 2357-2365. (https://doi.org/10.1210/ endo.143.6.8836)

Mullany LK, Rohira AD, Leach JP, Kim JH, Monroe TO, Ortiz AR, Stork B, Gaber MW, Sarkar P, Sikora AG, et al. 2020 A steroid receptor coactivator stimulator (MCB-613) attenuates adverse remodeling after myocardial infarction. PNAS 117 31353-31364. (https://doi. org/10.1073/PNAS.2011614117)

Orshal JM \& Khalil RA 2004 Gender, sex hormones, and vascular tone. American Journal of Physiology: Regulatory, Integrative and Comparative Physiology 286 R233-R249. (https://doi.org/10.1152/ajpregu.00338.2003)

Pawar S, Hantak AM, Bagchi IC \& Bagchi MK 2014 Minireview: steroidregulated paracrine mechanisms controlling implantation. Molecular Endocrinology 28 1408-1422. (https://doi.org/10.1210/me.2014-1074)

Ramathal CY, Bagchi IC, Taylor RN \& Bagchi MK 2010 Endometrial decidualization: of mice and men. Seminars in Reproductive Medicine $\mathbf{2 8}$ 17-26. (https://doi.org/10.1055/s-0029-1242989)

Rohira AD \& Lonard DM 2017 Steroid receptor coactivators present a unique opportunity for drug development in hormone-dependent cancers. Biochemical Pharmacology 140 1-7. (https://doi.org/10.1016/j. bcp.2017.04.005)

Sakakura T, Nishizuka Y \& Dawe CJ 1976 Mesenchyme-dependent morphogenesis and epithelium-specific cytodifferentiation in mouse mammary gland. Science 194 1439-1441. (https://doi.org/10.1126/ SCIENCE.827022)

Sever R \& Glass CK 2013 Signaling by nuclear receptors. Cold Spring Harbor Perspectives in Biology 5 a016709. (https://doi.org/10.1101/ CSHPERSPECT.A016709)

Smith RS, Smith TJ, Blieden TM \& Phipps RP 1997 Fibroblasts as sentinel cells. Synthesis of chemokines and regulation of inflammation. American Journal of Pathology 151 317-322.

Song X, Chen J, Zhao M, Zhang C, Yu Y, Lonard DM, Chow DC, Palzkill T, $\mathrm{Xu}$ J, O'Malley BW, et al. 2016 Development of potent small-molecule inhibitors to drug the undruggable steroid receptor coactivator-3. PNAS 113 4970-4975. (https://doi.org/10.1073/pnas.1604274113)

Song X, Chen H, Zhang C, Yu Y, Chen Z, Liang H, Van Buren G, McElhany AL, Fisher WE, Lonard DM, et al. 2019 SRC-3 inhibition blocks tumor growth of pancreatic ductal adenocarcinoma. Cancer Letters 442 310-319. (https://doi.org/10.1016/j.canlet.2018.11.012)

Suh JH, Lai L, Nam D, Kim J, Jo J, Taffet GE, Kim E, Kaelber JT, Lee HK, Entman ML, et al. 2017 Steroid receptor coactivator-2 (SRC-2) coordinates cardiomyocyte paracrine signaling to promote pressure overload-induced angiogenesis. Journal of Biological Chemistry 292 21643-21652. (https://doi.org/10.1074/jbc.M117.804740)

Szwarc MM, Kommagani R, Lessey BA \& Lydon JP 2014 The p160/steroid receptor coactivator family: potent arbiters of uterine physiology and dysfunction1. Biology of Reproduction 91 122-122. (https://doi. org/10.1095/biolreprod.114.125021)

Szwarc MM, Hai L, Gibbons WE, White LD, Mo Q, Kommagani R, Lanz RB, DeMayo FJ, O'Malley BW \& Lydon JP 2018 Retinoid signaling controlled by SRC-2 in decidualization revealed by transcriptomics. Reproduction 156 387-395. (https://doi.org/10.1530/ REP-18-0282)

Turner MD, Nedjai B, Hurst T \& Pennington DJ 2014 Cytokines and chemokines: at the crossroads of cell signalling and inflammatory disease. Biochimica et Biophysica Acta 1843 2563-2582. (https://doi. org/10.1016/j.bbamcr.2014.05.014)

Van Linthout S, Miteva K \& Tschöpe C 2014 Crosstalk between fibroblasts and inflammatory cells. Cardiovascular Research 102 258-269. (https:// doi.org/10.1093/cvr/cvu062)

Wang W, Bian K, Vallabhaneni S, Zhang B, Wu RC, O'Malley BW \& Long W 2014 ERK3 promotes endothelial cell functions by upregulating SRC-3/SP1-mediated VEGFR2 expression. Journal of Cellular Physiology 229 1529-1537. (https://doi.org/10.1002/ jcp.24596)

White RE 2002 Estrogen and vascular function. Vascular Pharmacology 38 73-80. (https://doi.org/10.1016/S0306-3623(02)00129-5)

Wu T \& Dai Y 2017 Tumor microenvironment and therapeutic response. Cancer Letters 387 61-68. (https://doi.org/10.1016/J. CANLET.2016.01.043)

Wynn TA 2008 Cellular and molecular mechanisms of fibrosis. Journal of Pathology 214 199-210. (https://doi.org/10.1002/path.2277)

Xu J, Qiu Y, DeMayo FJ, Tsai SY, Tsai MJ \& O’Malley BW 1998 Partial hormone resistance in mice with disruption of the steroid receptor coactivator-1 (SRC-1) gene. Science 279 1922-1925. (https://doi. org/10.1126/science.279.5358.1922)

Xu J, Wu RC \& O'Malley BW 2009 Normal and cancer-related functions of the p160 steroid receptor co-activator (SRC) family. Nature Reviews: Cancer 9 615-630. (https://doi.org/10.1038/nrc2695)

York B, Reineke EL, Sagen JV, Nikolai BC, Zhou S, Louet JF, Chopra AR, Chen X, Reed G, Noebels J, et al. 2012 Ablation of steroid receptor coactivator-3 resembles the human CACT metabolic myopathy. Cell Metabolism 15 752-763. (https://doi.org/10.1016/j.cmet.2012.03.020)

Yu C, York B, Wang S, Feng Q, Xu J \& O'Malley BW 2007 An essential function of the SRC-3 coactivator in suppression of cytokine mRNA translation and inflammatory response. Molecular Cell 25 765-778. (https://doi.org/10.1016/J.MOLCEL.2007.01.025)

Yuan Y \& Xu J 2007 Loss-of-function deletion of the steroid receptor coactivator-1 gene in mice reduces estrogen effect on the vascular injury response. Arteriosclerosis, Thrombosis, and Vascular Biology 27 1521-1527. (https://doi.org/10.1161/ATVBAHA.107.144477)

Yuan Y, Liao L, Tulis DA \& Xu J 2002 Steroid receptor coactivator-3 is required for inhibition of neointima formation by estrogen. Circulation 105 2653-2659. (https://doi.org/10.1161/01. CIR.0000018947.95555.65)

Zhang Y \& Shi W 2019 Steroid receptor coactivator-1 regulates glioma angiogenesis through polyomavirus enhancer activator 3 signaling. Biochemistry and Cell Biology 97 488-496. (https://doi.org/10.1139/ bcb-2018-0114)

Zhang X, Ibrahimi OA, Olsen SK, Umemori H, Mohammadi M \& Ornitz DM 2006 Receptor specificity of the fibroblast growth factor family. The complete mammalian FGF family. Journal of Biological Chemistry 281 15694-15700. (https://doi.org/10.1074/jbc. M601252200)

Received in final form 3 December 2020

Accepted 16 December 2020

Accepted Manuscript published online 19 December 2020 https://joe.bioscientifica.com https://doi.org/10.1530/JOE-20-0511 (c) 2021 Society for Endocrinology Published by Bioscientifica Ltd. Printed in Great Britain 